Research Paper

\title{
Incidence of Ipilimumab-Related Serious Adverse Events in Patients with Advanced Cancer: A Meta-Analysis
}

\author{
Chang-Ying Guo ${ }^{1,2,3^{*}}$, Si-Cong Jiang $3^{3^{*}}$, Yu-Kang Kuang1, Hao Hu1 ${ }^{\bowtie}$ \\ 1. Department of Thoracic Surgery, Jiangxi Cancer Hospital, People's Republic of China \\ 2. Department of Thoracic Surgery, Ji' an Central Hosipital, People's Republic of China. \\ 3. Department of Thoracic Surgery, Medical College of Nanchang University, People's Republic of China. \\ *Chang-Ying Guo and Si-Cong Jiang contributed equally to this work. \\ $\triangle$ Corresponding author: Hao Hu, Department of Thoracic Surgery, Jiangxi Cancer Hospital, No. 519 Beijing East Road, Nanchang, 330029, China. (E-mail: \\ qianhe89513@163.com) \\ (c) Ivyspring International Publisher. This is an open access article distributed under the terms of the Creative Commons Attribution (CC BY-NC) license \\ (https://creativecommons.org/licenses/by-nc/4.0/). See http://ivyspring.com/terms for full terms and conditions.
}

Received: 2018.06.26; Accepted: 2018.10.27; Published: 2019.01.01

\begin{abstract}
Background: Little is known about the incidence of ipilimumab-related serious adverse events (SAEs) across various tumor types, drug doses and treatment regimens.

Methods: PubMed database was searched up to November, 2017 to identify prospective clinical trials of ipilimumab therapy for adult patients with cancer. Comparisons of the incidence were based on the $\chi^{2}$ test in univariate analysis and the logistic regression model in multivariate analysis.

Results: Twenty-four studies (4549 patients) with 35 independent study cohorts ( 21 melanoma, 6 prostate cancer, 5 NSCLC, and 3 SCLC cohorts) of ipilimumab were included in the meta-analysis. The overall incidence of SAEs during ipilimumab mono-therapy was $26.1 \%(95 \% \mathrm{Cl}, 21.1 \%-31.8 \%)$. SAEs were more frequent in the $10 \mathrm{mg} / \mathrm{kg}$ groups than in the $3 \mathrm{mg} / \mathrm{kg}$ groups $(35.9 \%$ vs $17.3 \%$; $\mathrm{P}$ $0.001)$. Combination therapy showed significantly higher incidence than mono-therapy in melanoma (33.8\% vs $25.0 \%$; $P=0.002$ ). After adjustment for potential confounders, multivariable analyses demonstrated lower odds of SAEs in NSCLC (odds ratio [OR] 0.52, 95\% Cl 0.40-0.69, P<0.001) and SCLC (OR 0.41, 95\% Cl 0.31-0.54, P < 0.001) compared with melanoma. The 10mg/kg cohort presented significantly higher odds than the $3 \mathrm{mg} / \mathrm{kg}$ (OR $2.84,95 \% \mathrm{Cl} 2.35-3.43, \mathrm{P}<0.001$ ). The combination therapy showed significantly higher odds than the mono-therapy $(\mathrm{OR} 1.38,95 \% \mathrm{Cl}$ $1.11-1.71, P=0.003)$.

Conclusions: The incidence of ipilimumab-related SAEs was higher in melanoma, the $10 \mathrm{mg} / \mathrm{kg}$ group and during combination therapy. Clinicians should enhance awareness of these risk factors in clinical practice and carefully monitor patients.
\end{abstract}

Key words: incidence, ipilimumab, serious adverse events, meta-analysis, risk factors

\section{Introduction}

Immune surveillance can recognize and eradicate tumor cells by the immune system [1]. Nevertheless, in the interaction of the host immune system with tumor cells, tumors have the ability to thwart effective immune surveillance through the up-regulation of negative immunologic regulators such as cytotoxic $\mathrm{T}$ lymphocyte-associated antigen 4 (CTLA-4) in tumor cells [2]. As demonstrated by antitumor immunity of ipilimumab (an anti-CTLA-4 antibody) in clinical trials, it was approved by US Food and Drug Administration for treatment of advanced melanoma in 2011[3]. Subsequently, combination therapy of ipilimumab with a programmed cell death 1 (PD-1) inhibitors, nivolumab, has also been approved as a treatment option for melanoma [4, 5]. Because of these regulatory approvals, it comes into the new era of rapidly expanding access and widespread prescription in the clinical practice. 
Meanwhile, several combination strategies that include ipilimumab with chemotherapy, radiotherapy, targeted therapy and other therapies are gradually being explored [6, 7]. A key issue for successful combinatorial therapies is whether intensified anti-tumor response and improved overall survival can be achieved without a corresponding additive or synergistic serious toxicity.

Ipilimumab-related adverse events, including dermatologic, gastrointestinal, hepatic, and endocrine events, were most commonly reported in clinical trials [8]. These adverse events are typically low grade and manageable within a relatively short time with proper management during the treatment [9]. Hence, they are frequently explained to the patients prior to ipilimumab treatment in the clinical setting or clinical trials. Although several reports have documented ipilimumab-related serious adverse events (SAEs), which leads not only to treatment interruption, discontinuation or hospitalizations but also to disabilities, life-threatening and deaths, the incidence of SAEs is generally ignored during shared decision-making process due to, partly, lack of data.

Currently, there has been no report of a systematic review or meta-analysis focusing on the incidence of ipilimumab-related SAEs across different tumor types, drug doses and between mono-therapy vs combination therapies. Because of the relatively small sample size commonly seen in clinical trials of ipilimumab, the information on SAEs based on the individual cohort data is limited. Given the increasing number of published trial results concerning ipilimumab, such a study would enable better patient management of these serious toxicities and properly judge the risk-benefit balance. It would also inform the design of future ipilimumab trials especially where combination approaches are being evaluated and where increased toxicity is key question to their success. In this scenario, we undertook a meta-analysis of prospective trials of ipilimumab in patients with cancer to determine and compare the incidence of SAEs based on tumor type, drug dose and mono-therapy vs combination therapy.

\section{Methods}

\section{Search Strategy and Study Selection}

A computerized search of citations from inception to November 27, 2017 was conducted using the PubMed database to identify prospective clinical trials of ipilimumab therapy (including mono-therapy and combination therapy) for adult patients with cancer. The following keywords were used for the search: ipilimumab and cancer (detailed search strategies are reported in Table S1). We also manually examined latest FDA drug label for ipilimumab and reviewed the reference lists of identified reports to determine additional pertinent information. After the titles and abstracts were reviewed, the full texts from potentially relevant publications were retrieved to satisfy the predefined eligibility criteria. Only the most complete, recent, and updated studies were included in case duplicate reports were identified. Meeting abstracts or oral presentations without published full-text original articles were not eligible for this study. All procedures were performed by 2 independent reviewers and confirmed by a third reviewer following Preferred Reporting Items for Systematic Reviews and Meta-Analyses guidelines [10].

\section{Data Extraction}

The SAE definition is based on currently accepted criteria in cancer clinical trials, including an adverse events leading to death, life-threatening condition, hospitalization or prolongation of hospitalization, disability or permanent damage, congenital anomaly or birth defect, requiring intervention to prevent permanent impairment or damage, or any other adverse events that may jeopardize the patient and may require medical or surgical intervention (treatment) to prevent one of the other outcomes [11, 12]. The data regarding ipilimumab-related SAEs was depended on the decision of the primary investigators in the original trials and/or updated in ClinicalTrials.gov. The study name, phase stage, tumor type, treatment regimens, ipilimumab dose, median age, the number of treated patients and the number of patients with treatment-related SAEs were extracted from the included studies. We also made efforts to contact study authors and the manufacturer of ipilimumab when relevant data were indeterminable.

\section{Study Endpoints}

The primary endpoints evaluated were: 1 . overall incidences of SAEs with ipilimumab monotherapy and combination therapy, respectively; 2 . comparisons of overall incidence rates across different tumor types, drug doses or mono-therapy vs combination therapy; 3 . risk factors of SAEs.

\section{Statistical Analysis}

All the statistical analyses were carried out using the Comprehensive Meta-analysis statistical software (Version 2, Biostat, Englewood, New Jersey) or SPSS 22.0. The rate of patients with SAEs and 95\% confidence interval (CI) were derived for each trial. The Cochrane Q-statistic was performed to assess statistical heterogeneity [13] and $\mathrm{I}^{2}$ statistic $[100 \times(\mathrm{Q}-$ 
df)/Q] was used to estimate the extent of variability attributable to statistical heterogeneity in the results between the included studies [14]. The pooled effect sizes were calculated using random-effects or fixed-effects model based on the heterogeneity across studies. When the assumption of homogeneity was deemed invalid (the Cochran $\mathrm{Q}$ statistic $\mathrm{P}<0.1$ ) [15] and a random-effects model was used. In this context, a subgroup analysis was explored to study the causes of heterogeneity. Otherwise, a fixed-effects model was applied. Comparisons of the incidence were based on the $\chi^{2}$ test in univariate analysis. A multivariate logistic regression model was conducted to assess the association between SAEs and clinical variables using odds ratio (OR) and corresponding 95\% CI. All five independent variables were included in each model: tumor type, drug dose, mono-therapy vs combination therapy, median age and trial phase. The patients were divided into younger and older groups with a median age cut-off of 65 years. We applied a funnel plot method together with the Egger's test [16] and Begg's test [17] to detect the possibility of publication bias among the trials. The Holm-Bonferroni method [18] was used to adjust reported P values and 95\% CIs for multiple significance testing to preserve overall type I error rates of 0.05 .

\section{Results}

\section{Eligible Studies and Main Characteristics}

A total of 1830 publications were retrieved through initial searches of electronic databases. After screening titles/abstracts of the studies, 76 studies were selected based on relevance to the research subject. After further reviewing the full-text of these potentially eligible articles, a total of 28 eligible studies ( $\mathrm{n}=4993$ patients) meeting the inclusion criteria were identified for the final analysis (Figure 1) $[4,19-45]$. The tumor types were widely distributed in these studies (18 studies in melanoma, four in non-small-cell lung cancer (NSCLC), three in prostate cancer, two in small-cell lung cancer (SCLC) and one in gastric or gastro-esophageal junction cancer). Of these, ten studies $[4,21,24,25,27,28,30,37,40,42]$ reported 2 treatment arms, one [23] reported 3 and one [29] reported 5 treatment arms. These cohorts were recorded separately, resulting in a total of 44 independent cohorts from the 28 studies for a systematic review (Table 1).

These 28 articles were evaluated for inclusion in the meta-analysis, which was primarily designed to compare the incidence of SAEs among different tumor types, drug doses, and between the groups treated with mono-therapy vs combination therapy during ipilimumab treatment. Study in gastric or gastro- esophageal junction cancer [38] was removed because of only 1 study with small sample sizes. Two studies $[4,20]$ of multiple drug doses were excluded because they included multiple drug doses, and single drug dose of the patients with SAE was always not available. Moreover, one study (two cohorts in 1 $\mathrm{mg} / \mathrm{kg}$ ) [42] and two independent cohorts $(0.3$ and 5 $\mathrm{mg} / \mathrm{kg}$ ) from corresponding two studies [23, 29] were not integrated because of small sample sizes for each cohort. In addition, an independent cohort was removed from one study [21] because of ipilimumab administered with prophylactic budesonide. Finally, 24 studies ( $\mathrm{n}=4549$ patients) [19, 21-37, 39-41, 43-45] with 35 independent cohorts ( 21 melanoma, 6 prostate cancer, 5 NSCLC, and 3 SCLC cohorts) were included in the meta-analysis.

1830 Records identified relevant articles

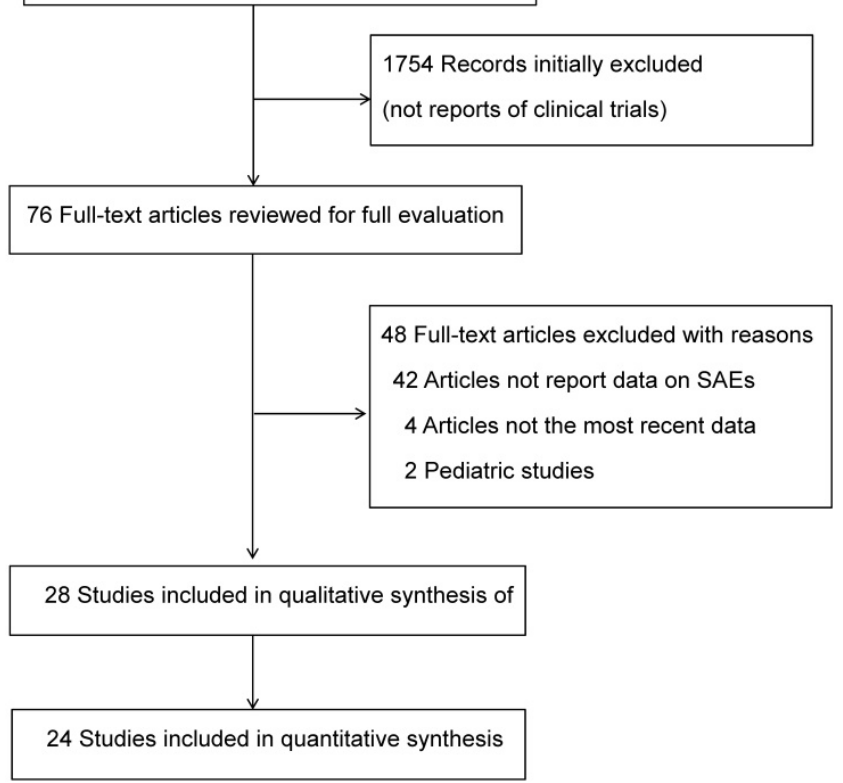

Figure 1. Flow Diagram of Study Inclusion. SAEs: Serious adverse events.

\section{Incidence of SAEs in the Systematic Review}

The incidence of SAEs of each study was listed in Table 1. The number of treated patients of each independent cohort ranged from 6 to 478. The incidence of SAEs ranged between $8.3 \%$ and $73.3 \%$, with the lowest incidence observed in the $0.3 \mathrm{mg} / \mathrm{kg}$ cohort of single-agent ipilimumab [23], and the highest in the phase II concurrent therapy with ipilimumab and dacarbazine terminated trial [32]. Of these 28 studies, 24 studies focusing on melanoma, prostate cancer, NSCLC, and SCLC with these two doses (3 or 10 $\mathrm{mg} / \mathrm{kg}$ ) were assessed in the meta-analysis.

\section{Incidence of SAEs in Patients Treated with Ipilimumab Mono-therapy}

A total of 2933 patients receiving ipilimumab 
mono-therapy in 14 studies (18 independent cohorts) $[19,21,22,24,29,31,34,37,39,40,43,44]$ with a focus on melanoma and prostate cancer were eligible for meta-analysis. The highest incidence $(45.9 \%$; $95 \% \mathrm{CI}$, $41.4 \%-50.4 \%$ ) was seen in a phase III melanoma trial with $10 \mathrm{mg} / \mathrm{kg}$ [34], and the lowest incidence (10.3\%; $95 \%$ CI, 3.9\%-24.3\%) was seen in a phase II melanoma trial with $3 \mathrm{mg} / \mathrm{kg}$ [25]. Meta-analysis showed the overall incidence of SAEs in patients receiving ipilimumab mono-therapy was $26.1 \% \quad(95 \% \quad \mathrm{CI}$, 21.1\%-31.8\%; heterogeneity test: $\mathrm{Q}=149.9 ; \mathrm{P}<0.001$; $\mathrm{I} 2=88.7$ ) (Figure 2). The incidence of SAEs during ipilimumab mono-therapy differed significantly with drug doses $(\mathrm{P}<0.001)$ but not with tumor types $(\mathrm{P}=$ 0.475) (Table 2). We have further determined the incidence separately according to their histology and drug dose to investigate the relationship between these factors and SAEs. As shown in Table 3, the incidence in the $10 \mathrm{mg} / \mathrm{kg}$ groups was significantly higher than in the $3 \mathrm{mg} / \mathrm{kg}$ groups $(35.9 \%$ versus $17.3 \% ; \mathrm{P}<0.001)$; however, it did not differ significantly between melanoma and prostate cancer $(\mathrm{P}=0.379)$.

Table 1. Incidence of ipilimumab-related serious adverse events in all studies included in the meta-analysis

\begin{tabular}{|c|c|c|c|c|c|c|c|}
\hline Study name and Year & Phase & Tumor type & Treatment regimens & $\begin{array}{l}\text { Dose } \\
(\mathrm{mg} / \mathrm{kg})\end{array}$ & $\begin{array}{l}\text { Median age } \\
\text { (rang, years) }\end{array}$ & $\begin{array}{l}\text { No. of } \\
\text { Patients a }\end{array}$ & $\begin{array}{l}\text { SAEs } \\
\text { No. }(\%)\end{array}$ \\
\hline Small et al, ${ }^{19} 2007$ & I & Prostate & Ipilimumab & 3 & $70(56-79)$ & 14 & $2(14.3)$ \\
\hline Weber et al, $^{20} 2008$ & $\mathrm{I} / \mathrm{II}$ & Melanoma & Ipilimumab & Multiple & $58(29-87)$ & 88 & $10(11.4)$ \\
\hline \multirow[t]{2}{*}{ Weber et al, ${ }^{21} 2009 \&$} & II & Melanoma & Ipilimumab & 10 & $61(26-86)$ & 57 & $21(36.8)$ \\
\hline & & & Ipilimumab+Prophylactic Budesonide & 10 & $58(30-82)$ & 58 & $26(44.8)$ \\
\hline O'Day et al,,22 2010 & II & Melanoma & Ipilimumab & 10 & $59(26-85)$ & 155 & 49 (31.6) \\
\hline \multirow[t]{3}{*}{ Wolchok et al,23 2010\& } & II & Melanoma & Ipilimumab『 & 0.3 & $59(25-85)$ & 72 & $6(8.3)$ \\
\hline & & & Ipilimumab & 3 & $59(29-78)$ & 71 & $13(18.3)$ \\
\hline & & & Ipilimumab & 10 & $56(19-83)$ & 71 & $19(26.8)$ \\
\hline \multirow[t]{2}{*}{ Hamid et al, ${ }^{24} 2010 \&$} & II & Melanoma & Ipilimumab & 3 & $54(23-78)$ & 40 & $7(17.5)$ \\
\hline & & & Ipilimumab & 10 & $56(26-87)$ & 42 & $8(19.0)$ \\
\hline \multirow[t]{2}{*}{ Hersh et al, ${ }^{25} 2011 \&$} & II & Melanoma & Ipilimumab & 3 & $66(25-82)$ & 39 & $4(10.3)$ \\
\hline & & & Ipilimumab+Dacarbazine & 3 & $60(27-82)$ & 35 & $5(14.3)$ \\
\hline Robert et al,26 2011 & III & Melanoma & Ipilimumab+Dacarbazine & 10 & 58 (NA) & 247 & $116(47.0)$ \\
\hline \multirow[t]{2}{*}{ Lynch et al, 27 2012\& } & II & NSCLC & Ipilimumab+Paclitaxel+Carboplatin (concurrent) & 10 & $59(36-82)$ & 71 & $20(28.2)$ \\
\hline & & & $\begin{array}{l}\text { Ipilimumab+Paclitaxel+Carboplatin } \\
\text { (phased) }\end{array}$ & 10 & $61(36-88)$ & 67 & $13(19.4)$ \\
\hline \multirow[t]{2}{*}{ Reck et al, $282013 \&$} & II & SCLC & Ipilimumab+Paclitaxel+Carboplatin (concurrent) & 10 & $57(44-80)$ & 42 & $10(23.8)$ \\
\hline & & & Ipilimumab+Paclitaxel+Carboplatin (phased) & 10 & $59(43-80)$ & 42 & $12(28.6)$ \\
\hline \multirow[t]{5}{*}{ Slovin et al,29 2013\& } & $\mathrm{I} / \mathrm{II}$ & Prostate & Ipilimumab & 3 & $69(55-78)$ & 8 & $2(25.0)$ \\
\hline & & & Ipilimumabף & 5 & $57(51-68)$ & 6 & $1(16.7)$ \\
\hline & & & Ipilimumab & 10 & $65(53-76)$ & 16 & $7(43.8)$ \\
\hline & & & Ipilimumab+Radiotherapy & 3 & $68(54-81)$ & 7 & $2(28.6)$ \\
\hline & & & Ipilimumab+Radiotherapy & 10 & $66(50-83)$ & 34 & $7(20.6)$ \\
\hline \multirow[t]{2}{*}{ Wolchok et al,4 2013 } & I & Melanoma & Ipilimumab+Nivolumab(concurrent) & Multiple & $58(22-79)$ & 53 & $26(49.1)$ \\
\hline & & & Ipilimumab+Nivolumab(sequenced) & Multiple & $64(23-89)$ & 33 & $7(21.2)$ \\
\hline \multirow{2}{*}{$\begin{array}{l}\text { Horinouchi et al, } \\
2014^{\&}\end{array}$} & I & NSCLC & Ipilimumab+Paclitaxel+Carboplatin (phased) & 3 & $62(27-65)$ & 8 & $3(37.5)$ \\
\hline & & & Ipilimumab+Paclitaxel+Carboplatin (phased) & 10 & $61(40-70)$ & 7 & $1(14.3)$ \\
\hline Yamazaki et al, ${ }^{31} 2015$ & II & Melanoma & Ipilimumab & 3 & $63(29-76)$ & 20 & $3(15.0)$ \\
\hline Yamazaki et al,32 2015 & II & Melanoma & Ipilimumab+Dacarbazine & 10 & $61(36-70)$ & 15 & $11(73.3)$ \\
\hline Amin et al, 332015 & II & Melanoma & Ipilimumab+Vemurafenib & 10 & 55 (NA) & 46 & $18(39.1)$ \\
\hline Eggermont et al, ${ }^{34} 2016$ & III & Melanoma & Ipilimumab & 10 & $51(20-84)$ & 471 & $216(45.9)$ \\
\hline Puzanov et al,35 2016 & I & Melanoma & Ipilimumab+Talimogene Laherparepvec & 3 & $61(29-84)$ & 19 & $4(21.1)$ \\
\hline Reck et al,36 2016 & III & SCLC & Ipilimumab+Etoposide+Platinum & 10 & $62(39-85)$ & 478 & $129(27.0)$ \\
\hline \multirow[t]{2}{*}{ Ascierto et al, ${ }^{37} 2016^{\&}$} & III & Melanoma & Ipilimumab & 3 & $62(51-71)$ & 362 & $66(18.2)$ \\
\hline & & & Ipilimumab & 10 & $62(49-70)$ & 364 & $133(36.5)$ \\
\hline Bang et al,38 2016 & II & G/GEJ & Ipilimumab & 10 & $65(34-86)$ & 57 & $17(29.8)$ \\
\hline Beer et al,39 2016 & III & Prostate & Ipilimumab & 10 & $70(44-91)$ & 399 & $135(33.8)$ \\
\hline \multirow[t]{2}{*}{ Chesney et al, ${ }^{40} 2017 \&$} & II & Melanoma & Ipilimumab & 3 & $64(23-90)$ & 95 & $15(15.8)$ \\
\hline & & & Ipilimumab+Talimogene Laherparepvec & 3 & $65(23-93)$ & 95 & $24(25.3)$ \\
\hline Govindan et al,41 2017 & III & NSCLC & Ipilimumab+Paclitaxel+Carboplatin & 10 & $64(28-84)$ & 388 & $129(33.2)$ \\
\hline \multirow[t]{2}{*}{ Hellmann et al,42 2017 } & I & NSCLC & Ipilimumab+Nivolumab(every-6-weeks) & 1 & $62(57-73)$ & 39 & $11(28.2)$ \\
\hline & & & Ipilimumab+Nivolumab(every-12-weeks) & 1 & $68(58-73)$ & 38 & $12(31.6)$ \\
\hline Schachter et al, ${ }^{43} 2017$ & III & Melanoma & Ipilimumab & 3 & $62(18-88)$ & 256 & $44(17.2)$ \\
\hline Weber et al,44 2017 & III & Melanoma & Ipilimumab & 10 & $54(18-86)$ & 453 & $183(40.4)$ \\
\hline Weide et al,45 2017 & II & Melanoma & Ipilimumab+interleukin-2 & 3 & 54 (NA) & 15 & $4(26.7)$ \\
\hline
\end{tabular}




\begin{tabular}{|c|c|c|c|c|}
\hline \multirow{2}{*}{$\frac{\text { Source }}{\text { Small et al, }{ }^{19} 2007}$} & Number of events & \multirow{2}{*}{$\frac{\text { Sample size }}{14}$} & \multirow{2}{*}{$\begin{array}{l}\begin{array}{l}\text { Incidence, \% } \\
\text { (95\%CI) }\end{array} \\
14.3(3.6-42.7)\end{array}$} & Incidence and $95 \% \mathrm{Cl}$ \\
\hline & 2 & & & \\
\hline Weber et al, ${ }^{21} 2009$ & 21 & 57 & $36.8(25.4-50.0)$ & \\
\hline O'Day et al, ${ }^{22} 2010$ & 49 & 155 & $31.6(24.8-39.3)$ & \\
\hline Wolchok et al, ${ }^{23} 2010^{\mathrm{a}}$ & 13 & 71 & $18.3(10.9-29.0)$ & \\
\hline Wolchok et al, ${ }^{23} 2010^{b}$ & 19 & 71 & $26.8(17.8-38.2)$ & \\
\hline Hamid et al, ${ }^{24} 2010^{\mathrm{a}}$ & 7 & 40 & $17.5(8.6-32.4)$ & \\
\hline Hamid et al, ${ }^{24} 2010^{b}$ & 8 & 42 & $19.0(9.8-33.7)$ & \\
\hline Hersh et al, ${ }^{25} 2011$ & 4 & 39 & $10.3(3.9-24.3)$ & \\
\hline Slovin et al, ${ }^{29} 2013^{a}$ & 2 & 8 & $25.0(6.3-62.3)$ & \\
\hline Slovin et al ${ }^{29} 2013^{b}$ & 7 & 16 & $43.8(22.5-67.6)$ & \\
\hline Yamazaki et al, ${ }^{31} 2015$ & 3 & 20 & $15.0(4.9-37.6)$ & \\
\hline Eggermont et al, ${ }^{34} 2016$ & 216 & 471 & $45.9(41.4-50.4)$ & \\
\hline Ascierto et al, ${ }^{37} 2016^{a}$ & 66 & 362 & $18.2(14.6-22.5)$ & \\
\hline Ascierto et al, ${ }^{37} 2016^{b}$ & 133 & 364 & $36.5(31.7-41.6)$ & \\
\hline Beer et al, ${ }^{39} 2016$ & 135 & 399 & $33.8(29.4-38.6)$ & \\
\hline Chesney et $\mathrm{al}^{40} 2017$ & 15 & 95 & $15.8(9.7-24.6)$ & 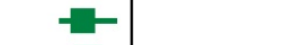 \\
\hline Schachter et al, ${ }^{43} 2017$ & 44 & 256 & $17.2(13.0-22.3)$ & + \\
\hline Weber et al, ${ }_{14}^{44} 2017$ & 183 & 453 & $40.4(36.0-45.0)$ & \\
\hline Overall & 927 & 2933 & $26.1(21.1-31.8)$ & \\
\hline \multicolumn{4}{|c|}{ Heterogeneity test: $Q=149.9 ; P<.001 ; I^{2}=88.7$} & 40 \\
\hline
\end{tabular}

Figure 2. Forest Plots of the Incidence of Serious Adverse Events During Ipilimumab Mono-therapy. $\mathrm{Cl}$ indicates confidence interval. a represents the study of 3 $\mathrm{mg} / \mathrm{kg}$ every 3 weeks. ${ }^{\circ}$ represents the study of $10 \mathrm{mg} / \mathrm{kg}$ every 3 weeks.

Table 2. Subgroup analysis in the incidence of ipilimumab-related SAEs

\begin{tabular}{|c|c|c|c|c|}
\hline & $\begin{array}{l}\text { Incidence, } \% \\
(95 \% \text { CI })\end{array}$ & $\begin{array}{l}\text { No. of } \\
\text { studies }\end{array}$ & $I^{2}$ & $\begin{array}{l}\text { P-value for } \\
\text { heterogeneity test }\end{array}$ \\
\hline \multicolumn{5}{|l|}{ Monotherapy } \\
\hline \multicolumn{5}{|l|}{ Tumor types } \\
\hline Melanoma & $25.0(19.4-31.6)$ & 14 & 91.1 & \multirow[b]{2}{*}{0.475} \\
\hline Prostate cancer & $30.9(17.7-48.3)$ & 4 & 3.6 & \\
\hline \multicolumn{5}{|l|}{ Drug dose } \\
\hline $3 \mathrm{mg} / \mathrm{kg}$ & $17.0(13.9-20.7)$ & 9 & 0 & \multirow[b]{2}{*}{$<0.001^{*}$} \\
\hline $10 \mathrm{mg} / \mathrm{kg}$ & $36.4(32.6-40.3)$ & 9 & 72.5 & \\
\hline \multicolumn{5}{|c|}{ Combination therapy } \\
\hline \multicolumn{5}{|l|}{ Tumor types } \\
\hline Melanoma & $34.3(25.5-44.4)$ & 6 & 80.6 & \multirow{4}{*}{0.582} \\
\hline Prostate cancer & $22.7(9.9-44.1)$ & 1 & 0 & \\
\hline NSCLC & $27.4(18.6-38.5)$ & 4 & 36.1 & \\
\hline SCLC & $26.5(17.0-38.9)$ & 2 & 0 & \\
\hline \multicolumn{5}{|l|}{ Drug dose } \\
\hline $3 \mathrm{mg} / \mathrm{kg}$ & $23.8(15.7-34.3)$ & 6 & 0 & \\
\hline $10 \mathrm{mg} / \mathrm{kg}$ & $31.7(25.7-38.3)$ & 11 & 79.9 & 0.189 \\
\hline
\end{tabular}

Abbreviations: SAEs, serious adverse events; NSCLC, non-small-cell lung cancer; SCLC, small-cell lung cancer. * Statistically significant difference.

\section{Incidence of SAEs in Patients Treated with Ipilimumab Combination therapy}

Data for SAEs was available for analysis from the total 1616 patients enrolled in the 11 studies (17 independent cohorts) [25-28, 30, 32, 33, 35, 36, 41, 45]. Combination regimens included chemotherapy (paclitaxel and carboplatin, etoposide and platinum, dacarbazine), radiotherapy, targeted therapy (vemurafenib) and others immunologic agents (talimogene laherparepvec or IL-2). The incidence of SAEs ranged from $14.3 \%$ to $73.3 \%$, with the highest incidence again noted in the phase II terminated trial of patients with melanoma [32], and the lowest incidence noted in the phase II trial of patients with NSCLC [30]. The overall incidence in the combination therapy group was 29.6\% (24.5\%-35.2\%; heterogeneity test: $\mathrm{Q}=58.1 ; \mathrm{P}<$ $0.001 ; \mathrm{I} 2=72.5$ ) (Figure 3 ). The incidence of SAEs during ipilimumab combination therapy did not varied significantly with tumor types $(\mathrm{P}=0.582)$, and drug doses $(\mathrm{P}=0.189)$. The pooled incidence rates in various tumor types in decreasing order were melanoma $(34.3 \%), \operatorname{NSCLC}(27.4 \%), \operatorname{SCLC}(26.5 \%)$ and prostate cancer $(22.7 \%)$ (Table 2). We have further assessed the relationship between these factors (histology and drug dose) and incidence of SAEs again. As shown in Table 3, SAEs were still more common with $10 \mathrm{mg} / \mathrm{kg}$ than with $3 \mathrm{mg} / \mathrm{kg}$ (31.6\% vs $23.9 \% ; \mathrm{P}=0.016)$. A lower incidence was detected among patients with NSCLC $(28.5 \%$ vs $33.8 \%$; $\mathrm{P}=$ $0.045)$ and SCLC $(26.9 \%$ vs $33.8 \% ; \mathrm{P}=0.003)$ than those with melanoma; however, the incidence still did not differ significantly between melanoma and prostate cancer.

\section{Incidence Between Mono-therapy vs Combination Therapy}

The incidence of SAEs during ipilimumab monotherapy vs combination therapy was compared in the studies [21, 22, 24-26, 31-35, 37, 40, 43-45] of melanoma because of no study of mono-therapy in patients with 
NSCLC/SCLC and the small sample sizes of patients with prostate cancer in the combination therapy group. The incidence was significantly higher in the combination therapy group than the mono-therapy group $(33.8 \%$ vs $25.0 \% ; \mathrm{P}=0.002)$.

Table 3. Incidence and odds ratio (OR) of SAEs for patients receiving ipilimumab among different tumor types and drug doses

\begin{tabular}{|c|c|c|c|c|c|}
\hline \multicolumn{6}{|c|}{ Patients treated with ipilimumab mono-therapy } \\
\hline & $\begin{array}{l}\text { Total no. of } \\
\text { patients treated }\end{array}$ & No. & $\begin{array}{l}\text { Incidence, } \\
\% \text { (95\% CI) }\end{array}$ & $\begin{array}{l}\text { OR }(95 \% \\
\text { CI) }\end{array}$ & $P$ value \\
\hline Overall & 2933 & 927 & $\begin{array}{l}26.1 \\
(21.1-31.8)\end{array}$ & & \\
\hline \multicolumn{6}{|l|}{ Tumor types } \\
\hline Melanoma & 2496 & 781 & $\begin{array}{l}25.0 \\
(19.3-31.6)\end{array}$ & Reference & 0.379 \\
\hline Prostate cancer & 437 & 146 & $\begin{array}{l}33.5 \\
(28.0-39.4)\end{array}$ & $\begin{array}{l}1.10 \\
(0.89-1.37)\end{array}$ & \\
\hline \multicolumn{6}{|l|}{ Drug doses } \\
\hline $3 \mathrm{mg} / \mathrm{kg}$ & 905 & 156 & $\begin{array}{l}17.3 \\
(15.0-19.9)\end{array}$ & Reference & $<0.001^{*}$ \\
\hline $10 \mathrm{mg} / \mathrm{kg}$ & 2028 & 771 & $\begin{array}{l}35.9 \\
(31.5-40.6)\end{array}$ & $\begin{array}{l}2.95 \\
(2.43-3.58)\end{array}$ & \\
\hline \multicolumn{6}{|c|}{ Patients treated with ipilimumab combination therapy } \\
\hline & $\begin{array}{l}\text { Total no. of } \\
\text { patients treated }\end{array}$ & No. & $\begin{array}{l}\text { Incidence, } \\
\%(95 \% \mathrm{CI})\end{array}$ & $\begin{array}{l}\text { OR }(95 \% \\
\text { CI) }\end{array}$ & $P$ value \\
\hline Overall & 1616 & 508 & $\begin{array}{l}29.6 \\
(24.5-35.2)\end{array}$ & & \\
\hline \multicolumn{6}{|l|}{ Tumor types } \\
\hline Melanoma & 472 & 182 & $\begin{array}{l}33.8 \\
(22.6-47.2)\end{array}$ & Reference & \\
\hline Prostate cancer & 41 & 9 & $\begin{array}{l}22.1 \\
(11.9-37.3)\end{array}$ & $\begin{array}{l}0.45 \\
(0.17-1.22)\end{array}$ & 0.112 \\
\hline NSCLC & 541 & 166 & $\begin{array}{l}28.5 \\
(22.2-35.8)\end{array}$ & $\begin{array}{l}0.71 \\
(0.50-0.99)\end{array}$ & $0.045^{*}$ \\
\hline SCLC & 562 & 151 & $\begin{array}{l}26.9 \\
(23.4-30.7)\end{array}$ & $\begin{array}{l}0.59 \\
(0.41-0.83)\end{array}$ & $0.003^{*}$ \\
\hline \multicolumn{6}{|l|}{ Drug doses } \\
\hline $3 \mathrm{mg} / \mathrm{kg}$ & 179 & 42 & $\begin{array}{l}23.9 \\
(18.2-30.8)\end{array}$ & Reference & \\
\hline $10 \mathrm{mg} / \mathrm{kg}$ & 1437 & 466 & $\begin{array}{l}31.6 \\
(25.4-38.6)\end{array}$ & $\begin{array}{l}1.57 \\
(1.09-2.25)\end{array}$ & $0.016^{*}$ \\
\hline
\end{tabular}

Abbreviations: SAEs, serious adverse events; NSCLC, non-small-cell lung cancer; SCLC, small-cell lung cancer. *Statistically significant difference. The incidence and OR were calculated from the trials included in this study by meta-analysis as described in the "Methods" section.

\section{Multivariable analysis}

A multivariate logistic regression model for the risk of SAEs occurrences was constructed using tumor type, drug dose $(10 \mathrm{mg} / \mathrm{kg}$ versus $3 \mathrm{mg} / \mathrm{kg})$, therapeutic regimens (combination therapy versus monotherapy), trial phases (phase III versus equivalent or lower than phase II) and median age (older versus younger). The results of multivariable analyses were displayed in Table 4 . On the bias of multivariate logistic regression model, there were significantly lower risk for SAEs among patients with NSCLC (OR $0.52,95 \%$ CI $0.40-0.69, \mathrm{P}<0.001$ ) and SCLC (OR 0.41, $95 \%$ CI $0.31-0.54, \mathrm{P}<0.001)$ than among those with melanoma; a slightly higher likelihood of SAEs was occurred in patients with NSCLC compared with those with SCLC (OR, 1.27, 95\% CI, 0.98-1.65; P =
0.076) despite not statistically significant. The odds of experiencing SAEs were not significantly different between patients with prostate cancer and melanoma, as well as patients with prostate cancer and NSCLC/SCLC. Patients in the $10 \mathrm{mg} / \mathrm{kg}$ group significantly more likely to experience SAEs than those in the $3 \mathrm{mg} / \mathrm{kg}$ group (OR 2.84, 95\% CI 2.35-3.43, $\mathrm{P}<0.001)$.

Combination therapy showed increased odds for the development of SAEs compared with mono-therapy group (OR 1.38, 95\% CI 1.11-1.71, P = 0.003). To further explore these effects, the combination therapy group was subdivided into the ipilimumab plus chemotherapy group and then ipilimumab plus others immunologic agents group because the number of patients in the ipilimumab plus radiotherapy/targeted therapy studies was small. Ipilimumab plus chemotherapy group showed significantly higher odds than the mono-therapy group (OR 1.38, 95\% CI 1.08-1.78, P = 0.011), so did ipilimumab plus others immunologic agents group (OR 2.12, 95\% CI 1.13-3.98, P = 0.019). However, a significant difference between these two combination therapy groups was not observed.

Table 4. Multivariable analyses results for the incidence of ipilimumab-related serious adverse events

\begin{tabular}{|c|c|c|}
\hline Variable & $\begin{array}{l}\text { Odds Ratio } \\
(95 \% \text { CI })\end{array}$ & $\begin{array}{l}P \\
\text { Value }\end{array}$ \\
\hline \multicolumn{3}{|l|}{ Tumor types } \\
\hline $\begin{array}{l}\text { Prostate cancer ( } 6 \text { studies, } 478 \text { patients) vs } \\
\text { melanoma ( } 21 \text { studies, } 2968 \text { patients) }\end{array}$ & $0.63(0.37-1.08)$ & 0.091 \\
\hline $\begin{array}{l}\text { SCLC ( } 3 \text { studies, } 562 \text { patients) vs melanoma ( } 21 \\
\text { studies, } 2968 \text { patients) }\end{array}$ & $0.41(0.31-0.54)$ & $<0.001^{*}$ \\
\hline $\begin{array}{l}\text { NSCLC ( } 5 \text { studies, } 541 \text { patients) vs melanoma ( } 21 \\
\text { studies, } 2968 \text { patients) }\end{array}$ & $0.52(0.40-0.69)$ & $<0.001^{*}$ \\
\hline $\begin{array}{l}\text { NSCLC ( } 5 \text { studies, } 541 \text { patients) vs SCLC ( } 3 \\
\text { studies, } 562 \text { patients) }\end{array}$ & $1.27(0.98-1.65)$ & 0.076 \\
\hline $\begin{array}{l}\text { Prostate cancer ( } 6 \text { studies, } 478 \text { patients) vs SCLC( } 3 \\
\text { studies, } 562 \text { patients) }\end{array}$ & $1.54(0.82-2.86)$ & 0.177 \\
\hline $\begin{array}{l}\text { Prostate cancer ( } 6 \text { studies, } 478 \text { patients) vs NSCLC } \\
\text { ( } 5 \text { studies, } 541 \text { patients) }\end{array}$ & $1.21(0.65-2.26)$ & 0.547 \\
\hline \multicolumn{3}{|l|}{ Drug doses } \\
\hline $10 \mathrm{mg} / \mathrm{kg}$ vs $3 \mathrm{mg} / \mathrm{kg}$ & $2.84(2.35-3.43)$ & $<0.001^{*}$ \\
\hline \multicolumn{3}{|l|}{ Therapeutic regimens } \\
\hline $\begin{array}{l}\text { Combination therapy ( } 17 \text { studies, } 1616 \text { patients) } \\
\text { vs mono-therapy (18 studies, } 2933 \text { patients) }\end{array}$ & $1.38(1.11-1.71)$ & $0.003^{*}$ \\
\hline $\begin{array}{l}\text { Ipilimumab plus chemotherapy (11studies, } 1400 \\
\text { patients) vs mono-therapy (18 studies, } 2933 \\
\text { patients) }\end{array}$ & $1.38(1.08-1.78)$ & $0.011^{*}$ \\
\hline $\begin{array}{l}\text { Ipilimumab plus others immunologic agents ( } 3 \\
\text { studies, } 129 \text { patients) vs mono-therapy }\end{array}$ & $2.12(1.13-3.98)$ & $0.019^{*}$ \\
\hline $\begin{array}{l}\text { Ipilimumab plus others immunologic agents ( } 3 \\
\text { studies, } 129 \text { patients) vs ipilimumab plus } \\
\text { chemotherapy (11studies, } 1400 \text { patients) }\end{array}$ & $1.54(0.78-3.02)$ & 0.214 \\
\hline \multicolumn{3}{|l|}{ Phase stage } \\
\hline $\begin{array}{l}\text { Phase III (9 studies, } 3418 \text { patients) vs equivalent } \\
\text { or lower than phase II ( } 26 \text { studies, } 1131 \text { patients) }\end{array}$ & $1.37(1.16-1.61)$ & $<0.001^{*}$ \\
\hline \multicolumn{3}{|l|}{ Median age } \\
\hline $\begin{array}{l}\text { Older ( } 8 \text { studies, } 612 \text { patients) vs younger ( } 27 \\
\text { studies, } 3937 \text { patients) }\end{array}$ & $1.20(0.74-1.94)$ & 0.453 \\
\hline
\end{tabular}




\begin{tabular}{|c|c|c|c|c|}
\hline \multirow{2}{*}{$\begin{array}{l}\text { Source } \\
\text { Hersh et al, }{ }^{25} 2011\end{array}$} & Number of events & \multirow{2}{*}{$\frac{\text { Sample size }}{35}$} & \multirow{2}{*}{$\begin{array}{l}\begin{array}{l}\text { Incidence, \% } \\
\text { (95\%Cl) }\end{array} \\
14.3(6.1-30.0)\end{array}$} & Incidence and $95 \% \mathrm{Cl}$ \\
\hline & 5 & & & 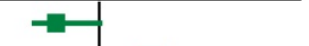 \\
\hline Robert et al, ${ }^{26} 2011$ & 116 & 247 & $47.0(40.8-53.2)$ & \\
\hline Lynch et al, ${ }^{27}$ 2012(con) & 20 & 71 & $28.2(19.0-39.7)$ & \\
\hline Lynch et al, ${ }^{27} 2012$ (phased) & 13 & 67 & $19.4(11.6-30.6)$ & \\
\hline Reck et al, ${ }^{28} 2013$ (con) & 10 & 42 & $23.8(13.3-38.9)$ & \\
\hline Reck et al, ${ }^{28} 2013$ (phased) & 12 & 42 & $28.6(17.0-43.9)$ & \\
\hline Slovin et al, ${ }^{29} 2013^{a}$ & 2 & 7 & $28.6(7.2-67.3)$ & \\
\hline Slovin et al, ${ }^{29} 2013^{b}$ & 7 & 34 & $20.6(10.1-37.3)$ & \\
\hline Horinouchi et al, ${ }^{30} 2014^{a}$ & 3 & 8 & $37.5(12.5-71.5)$ & \\
\hline Horinouchi et al ${ }^{30} 2014^{b}$ & 1 & 7 & $14.3(2.0-58.1)$ & \\
\hline Yamazaki et al, ${ }^{32} 2015$ & 11 & 15 & $73.3(46.7-89.6)$ & . \\
\hline Amin et al, ${ }^{33} 2015$ & 18 & 46 & $39.1(26.2-53.8)$ & \\
\hline Puzanov et al,$^{35} 2016$ & 4 & 19 & $21.1(8.1-44.6)$ & \\
\hline Reck et al, ${ }^{36} 2016$ & 129 & 478 & $27.0(23.2-31.1)$ & \\
\hline Chesney et al, ${ }^{40} 2017$ & 24 & 95 & $25.3(17.5-34.9)$ & \\
\hline Govindan et al, ${ }^{41} 2017$ & 129 & 388 & $33.2(28.7-38.1)$ & \\
\hline Weide et al, ${ }^{45} 2017$ & 4 & 15 & $26.7(10.4-53.3)$ & \\
\hline Overall & 508 & 1616 & $29.6(24.5-35.2)$ & \\
\hline Heterogeneity test: $Q=58.1 ;$ & $001 ; 1^{2}=72$ & & & $\begin{array}{c}50 \\
\text { Incidence, } \%\end{array}$ \\
\hline
\end{tabular}

Figure 3. Forest Plots of the Incidence of Serious Adverse Events During Ipilimumab Combination therapy. $\mathrm{Cl}$ indicates confidence interval. a represents the study of $3 \mathrm{mg} / \mathrm{kg}$ every 3 weeks. b represents the study of $10 \mathrm{mg} / \mathrm{kg}$ every 3 weeks.

Patients in phase III trials were significantly more likely to experience SAEs compared with patients in equivalent or lower than phase II trials (OR 1.37, 95\% CI 1.16-1.61, P < 0.001); however, no significant difference was found between younger and older patients.

Funnel plots along with the Egger's test and Begg's test were performed to evaluate the presence of publication bias. As shown in Funnel plots (Figure 4), there was minimal publication bias in the mono-therapy data. Additional tests to quantify publication bias were discordant (Egger test $\mathrm{P}=0.02$, Begg test $\mathrm{P}=0.29$ ). Therefore, the presence of publication bias in the mono-therapy data remains uncertain. However, publication bias was not evident in the combination data based on visual inspection of funnel plots (Figure 5) and additional quantitative tests (Egger test $P=0.39$, Begg test $P=0.51$ ).

\section{Discussion}

To our knowledge, the present study is the first meta-analysis with a focus on investigating the incidence of treatment-related SAEs during ipilimumab treatment in advanced cancer that compared the incidence among different tumor types, drug doses and treatment regimens. In the current meta-analysis of the published clinical trial results of ipilimumab therapy at a dose of 3 or $10 \mathrm{mg} / \mathrm{kg}$ for melanoma, prostate cancer, NSCLC, and SCLC, the overall incidence of ipilimumab-related SAEs was $26.1 \%$ for mono-therapy and $29.6 \%$ for combination therapy. The incidence was significantly higher in patients with melanoma than those with NSCLC and SCLC. The increased incidence was observed in $10 \mathrm{mg} / \mathrm{kg}$ groups than $3 \mathrm{mg} / \mathrm{kg}$ groups. Combination therapy was associated with a higher incidence compared with mono-therapy. No significant difference was found in the occurrence of SAEs between older and younger patients. These findings will help physicians and patients to recognize the incidence and risk of SAEs with the administration of ipilimumab in the clinical setting or clinical trials.

A higher incidence of SAEs was observed among patients with melanoma than patients with NSCLC and SCLC. While interesting, such findings are not surprising given that the response rates in patients treated with ipilimumab were distinct across different tumor types. Considering the proposed mechanism of action of ipilimumab, the differences in local tumor microenvironment, immune cell infiltrate especially T-cell, and adaptive immune response may generate the different toxicities [46-49]. Underlying disease, existing tumor burden, and prior therapies may also influence the differences. Furthermore, higher life expectancy in advanced melanoma patients may have influenced the higher rates of SAEs in these patients when treated with ipilimumab when compared with patients with advanced NSCLC and SCLC. One could argue that patients with melanoma could receive longer treatment durations and hence experience more SAEs. However, treatment duration was same (four doses) among those cohorts and thus the higher incidence of SAEs in patients with melanoma in this study may not be attributed to longer treatment 
durations. The incidence was still significantly higher for melanoma compared with NSCLC and SCLC in the multivariable analyses, adding further strength of evidence for this observation. Nevertheless, no significantly difference was seen in the incidence of SAEs for patients with prostate cancer compared with patients with melanoma based on univariate and multivariable analyses. Currently the underlying reasons for these observations are unidentified and remain to be further investigated. These results demonstrate the different susceptibilities of developing ipilimumab-related SAEs among patients with different tumor types. Because the clinical trials always did not report the data on treatment-related SAEs according to system organ class, we could not compare the incidence of each system organ across tumor types separately. The identification of specific SAEs issues related to various tumor types will require joint efforts and large pooled individual patient data for analysis.
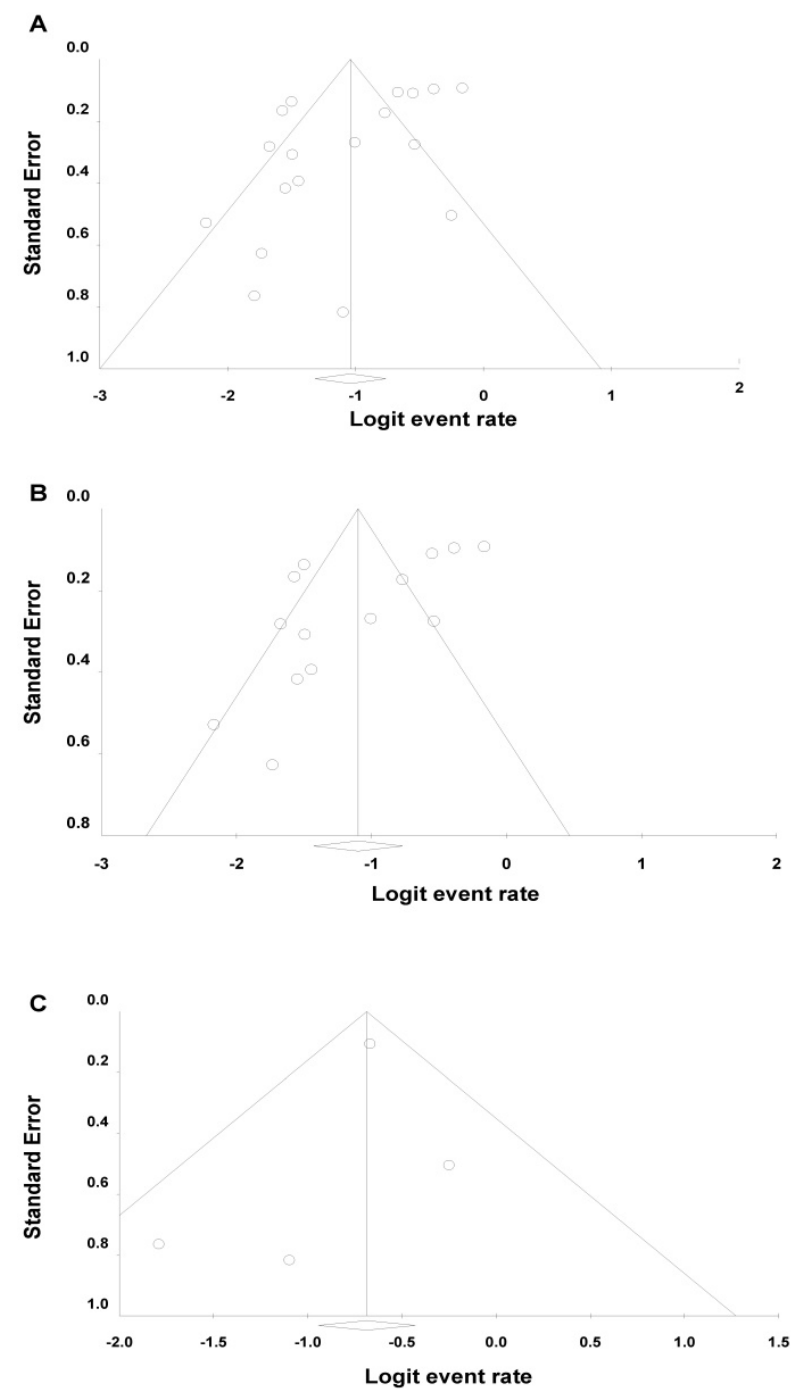

Figure 4. Funnel Plots for Ipilimumab Mono-therapy Studies for All Tumor Types (A), for Melanoma (B), and Prostate cancer (C).
Consistent with the reported phase III trial comparing 10 versus $3 \mathrm{mg} / \mathrm{kg}$ ipilimumab in metastatic melanoma [37], we found the incidence of SAEs was significantly higher in the $10 \mathrm{mg} / \mathrm{kg}$ group than $3 \mathrm{mg} / \mathrm{kg}$ group, suggesting the dose-dependent on SAEs. The incidence remained significantly higher for the $10 \mathrm{mg} / \mathrm{kg}$ group compared with $3 \mathrm{mg} / \mathrm{kg}$ group in the multivariable analyses after controlling for others factors, further demonstrating dose-effect relationship in the incidence of SAEs, with increased incidence associated with the high-dose of ipilimumab. This finding emphasizes a need for enhanced awareness and careful monitoring of the possibility of SAEs occurrence during high-dose ipilimumab therapy.
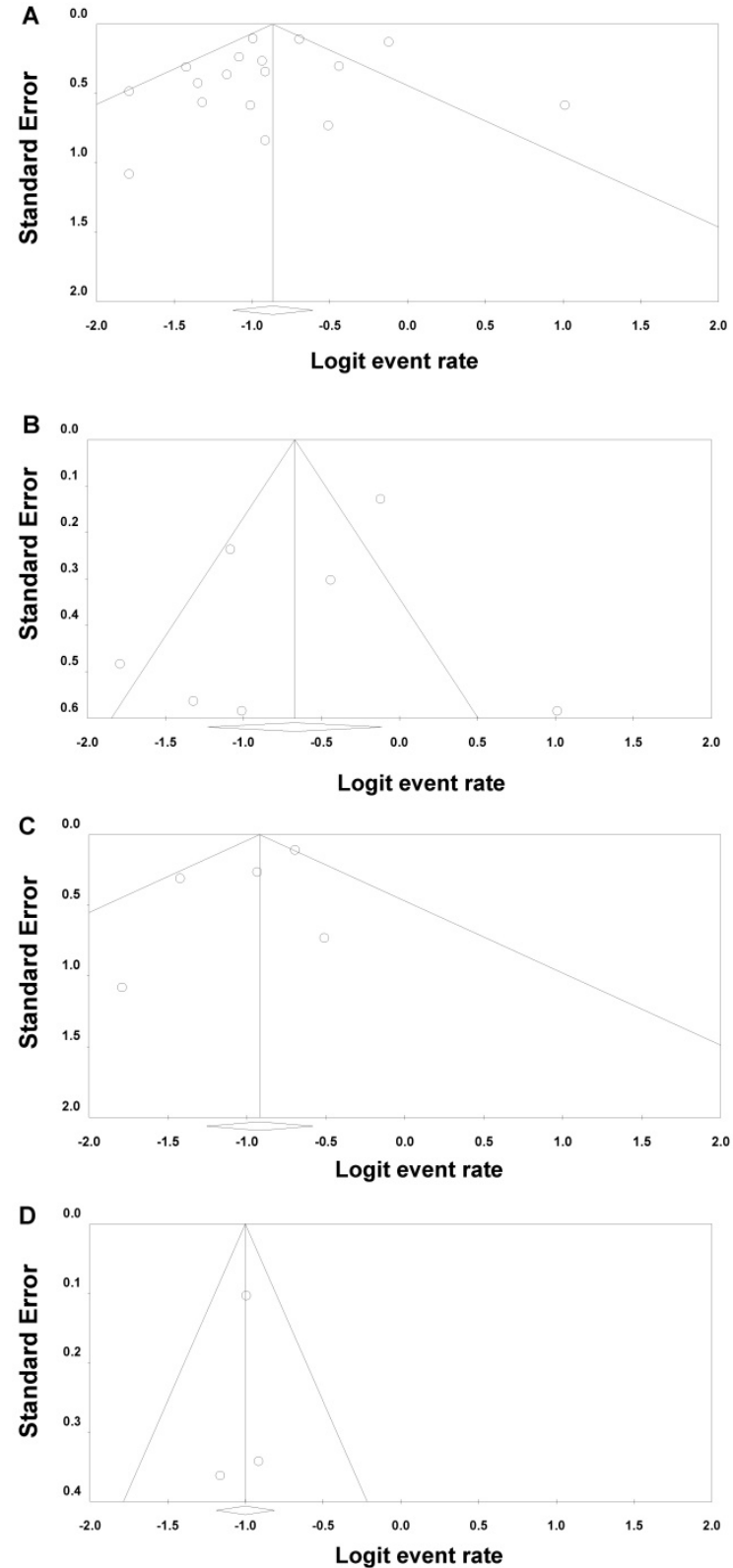

Figure 5. Funnel Plots for Ipilimumab Combination Studies for All Tumor Types (A), for Melanoma (B), NSCLC(C) and SCLC (D). 
The incidence of SAEs was significantly higher in the combination therapy cohort than mono-therapy cohort among patients with melanoma, indicating the additive effects of combination therapy on serious toxic effects. In the multivariable-adjusted analysis, we also detected a significantly higher incidence in studies investigating combination therapy than those that evaluated mono-therapy. This observation further demonstrates the synergistic effect of combination therapy on serious toxicities. When the combination therapy group was further subdivided the ipilimumab plus chemotherapy group and then ipilimumab plus others immunologic agents group, all results remained significant. The incidence of SAEs did not vary significantly between these two combination regimens; however, slightly higher odds and the wide variation in confidence intervals may indicate that the influences of various combination regimens on SAEs may be distinct. Although the detailed role and effect of chemotherapy and others immunologic agents on the development of SAEs when administered in combination with ipilimumab remain to be further evaluated, physicians should be alerted to the significantly higher incidence of SAEs in patients receiving combination therapy and must be aware of possible signs and symptoms of serious toxicities during combined treatment.

The incidence of SAEs was higher in the phase III trials than in the equivalent or lower than phase II trials based on the multivariable analyses. It is possible that the larger sample sizes and maturation of the trial design in later-phase trials make SAEs easier to detect and recognize, highlighting the importance and necessity of conducting large-sample clinical trials. We did an exploratory investigation to identify whether there was a difference in the occurrence of these SAEs between older and younger patients, but failed. As documented in previous reports [50, 51], older patients presented a similar safety profiles compared to younger patients, again suggesting that older patients did not appear to compromise the SAEs during ipilimumab treatment. Thus, until more knowledge obtained, we do not suggest that age at cutoff values of 65 as a biomarker to select patients who derive higher odds of experiencing SAEs from ipilimumab therapy.

Although there has no meta-analysis estimating the incidence and risk factors of SAEs with ipilimumab, a few meta-analyses investigating others adverse events, especially immune-related adverse events (irAEs), were generally consistent with our findings. For example, the risk of developing irAEs was dependent of dosage, with higher incidence of irAEs being seen in high-dose of ipilimumab group [52]. Another study of immune checkpoint inhibitors (including CTLA-4 and PD-1/PD-L1 inhibitors ) showed that melanoma patients treated with PD-1 inhibitors had a higher frequency of selected irAEs compared with NSCLC [8]. However, a network meta-analysis [53] did not show a dose-dependent increase in all adverse events, partly, due to comparison was made among small number of patients. This highlights the importance and necessity of doing such a study in a large number of patients. Our current meta-analyses including 4,549 patients from prospective trials is thus the largest meta-analysis of ipilimumab-related adverse events in patients with advanced cancer and offers a reliable quality evidence for evaluating the risk-benefit balance during ipilimumab treatment. Our study is also the first meta-analysis to determine the incidence and risk factors of SAEs with any immune checkpoint inhibitors.

The present study has several limitations. First, the meta-analysis focused on melanoma, prostate cancer, NSCLC, and SCLC with these two doses (3 or $10 \mathrm{mg} / \mathrm{kg}$ ), and others aspects, such as study at a dose of $0.3,1,5 \mathrm{mg} / \mathrm{kg}$, were not included because of the limited number of published studies with small sample sizes. Further researches with larger number of studies in a larger variety of tumors, drug dose and therapeutic regimen may help to verify these findings. Second, SAEs were not primary endpoint of any of the trials in the included studies. The observed incidence had significant heterogeneity among the included studies might bias the results, which might reflect differences in sample sizes, drug doses, tumor types, and other factors among these studies. But the random-effects model was applied to estimate overall incidence may be able to minimize the problem in our analysis. Third, all the patients were selected with strict inclusion criteria and exclusion criteria in these studies and then the incidence of SAEs in patients with comorbidities and poor performance status in a real world population may be higher. Fourth, the study may have a potential publication bias despite it was not detectable by our investigation. Fifth, the unavailability of SAEs data from 42 studies (see Figure 1 and Table S2 in the Supplement) as well as the detection of significant heterogeneity observed in the meta-analysis of the incidence of SAEs are other limitations of our meta-analysis. Finally, the incidence calculation was trial-level estimates, and several important details about toxicities at the patient level cannot be properly assessed.

\section{Conclusions}

A higher incidence of ipilimumab-related SAEs was demonstrated in patients with melanoma and in patients treated with combination therapy. The 
present study has further showed the incidence of SAEs was dose-dependent, with increased rate in the high-dose cohort. It is important for clinicians and patients to understanding the risk-benefit ratio associated with ipilimumab therapy and to monitor closely to identify and treat SAEs. Identification of reliable biomarkers and risk factors to predict the occurrence of SAEs in patients is a crucial strategy to direct the rational use of ipilimumab as well as enable personalized-medicine. Future studies are needed to investigate the optimal prevention and management of SAEs associated with ipilimumab treatment.

\section{Abbreviations}

SAEs: serious adverse events; CTLA-4: cytotoxic T lymphocyte-associated antigen 4; PD-1: programmed cell death 1; NSCLC: non-small-cell lung cancer; SCLC: small-cell lung cancer; CI: confidence interval; OR: odds ratio.

\section{Supplementary Material}

Supplementary tables.

http://www.jcancer.org/v10p0120s1.pdf

\section{Acknowledgements}

This study was supported by grants from the Jiangxi Province's Program of the Preponderant Team Building in Science and Technology Innovation (NO. 20161BCB24011), the National Natural Science Foundation of China (NO. 81560382) and the Key Research and Development Program of Jiangxi Province (NO. 20181BBG70017).

\section{Competing Interests}

The authors have declared that no competing interest exists.

\section{References}

1. Hanahan D, Weinberg RA. Hallmarks of cancer: the next generation. Cell. 2011; 144: 646-74.

2. Chen DS, Mellman I. Oncology meets immunology: the cancer-immunity cycle. Immunity. 2013; 39: 1-10.

3. Hodi FS, O'Day SJ, McDermott DF, Weber RW, Sosman JA, Haanen JB, et al. Improved Survival with Ipilimumab in Patients with Metastatic Melanoma. N Engl J Med. 2010; 363: 711-23.

4. Wolchok JD, Kluger H, Callahan MK, Postow MA, Rizvi NA, Lesokhin AM, et al. Nivolumab plus ipilimumab in advanced melanoma. N Engl J Med. 2013; 369: 122-33.

5. Larkin J, Chiarion-Sileni V, Gonzalez R, Grob JJ, Cowey CL, Lao CD, et al. Combined Nivolumab and Ipilimumab or Monotherapy in Untreated Melanoma. N Engl J Med. 2015; 373: 23-34.

6. Mahoney KM, Rennert PD, Freeman GJ. Combination cancer immunotherapy and new immunomodulatory targets. Nat Rev Drug Discov. 2015; 14: 561-84.

7. Gotwals P, Cameron S, Cipolletta D, Cremasco V, Crystal A, Hewes B, et al. Prospects for combining targeted and conventional cancer therapy with immunotherapy. Nat Rev Cancer. 2017; 17: 286-301.

8. Khoja L, Day D, Wei-Wu Chen T, Siu LL, Hansen AR. Tumour- and class-specific patterns of immune-related adverse events of immune checkpoint inhibitors: a systematic review. Ann Oncol. 2017; 28: 2377-85.

9. Tarhini A. Immune-mediated adverse events associated with ipilimumab ctla- 4 blockade therapy: the underlying mechanisms and clinical management. Scientifica (Cairo). 2013; 2013: 857519.
10. Moher D, Liberati A, Tetzlaff J, Altman DG. Preferred reporting items for systematic reviews and meta-analyses: The PRISMA statement. International Journal of Surgery. 2010; 8: 336-41.

11. Gyawali B, Shimokata T, Ando M, Honda K, Ando Y. Risk of serious adverse events and fatal adverse events with sorafenib in patients with solid cancer: a meta-analysis of phase 3 randomized controlled trialsdagger. Ann Oncol. 2017; 28: 246-53.

12. Larkin J, Chmielowski B, Lao CD, Hodi FS, Sharfman W, Weber J, et al. Neurologic Serious Adverse Events Associated with Nivolumab Plus Ipilimumab or Nivolumab Alone in Advanced Melanoma, Including a Case Series of Encephalitis. Oncologist. 2017; 22: 709-18.

13. Cochran WG. Some methods of strengthening the common $\chi^{2}$ tests. Biometrics. 1954; 23.

14. Higgins JP, Thompson SG, Deeks JJ, Altman DG. Measuring inconsistency in meta-analyses. BMJ. 2003; 327: 557-60.

15. Lau J, Ioannidis JPA, Schmid CH. Quantitative Synthesis in Systematic Reviews. Ann Intern Med. 1997; 127: 820-6.

16. Egger M, Smith GD, Schneider M, Minder C. Bias in meta-analysis detected by a simple, graphical test. BMJ. 1997; 315: 629-34.

17. Begg CB, Mazumdar M. Operating characteristics of a rank correlation test for publication bias. Biometrics. 1994: 1088-101.

18. Holm S. A Simple Sequentially Rejective Multiple Test Procedure. Scandinavian Journal of Statistics. 1979; 6: 65-70.

19. Small EJ, Tchekmedyian NS, Rini BI, Fong L, Lowy I, Allison JP. A pilot trial of CTLA-4 blockade with human anti-CTLA-4 in patients with hormone-refractory prostate cancer. Clin Cancer Res. 2007; 13: 1810-5.

20. Weber JS, O'Day S, Urba W, Powderly J, Nichol G, Yellin M, et al. Phase I/II study of ipilimumab for patients with metastatic melanoma. J Clin Oncol. 2008; 26: 5950-6.

21. Weber J, Thompson JA, Hamid O, Minor D, Amin A, Ron I, et al. A randomized, double-blind, placebo-controlled, phase II study comparing the tolerability and efficacy of ipilimumab administered with or without prophylactic budesonide in patients with unresectable stage III or IV melanoma. Clin Cancer Res. 2009; 15: 5591-8.

22. O'Day SJ, Maio M, Chiarion-Sileni V, Gajewski TF, Pehamberger $H$, Bondarenko IN, et al. Efficacy and safety of ipilimumab monotherapy in patients with pretreated advanced melanoma: a multicenter single-arm phase II study. Ann Oncol. 2010; 21: 1712-7.

23. Wolchok JD, Neyns B, Linette G, Negrier S, Lutzky J, Thomas L, et al. Ipilimumab monotherapy in patients with pretreated advanced melanoma: a randomised, double-blind, multicentre, phase 2, dose-ranging study. Lancet Oncol. 2010; 11: 155-64.

24. Hamid O, Schmidt H, Nissan A, Ridolfi L, Aamdal S, Hansson J, et al. A prospective phase II trial exploring the association between tumor microenvironment biomarkers and clinical activity of ipilimumab in advanced melanoma. J Transl Med. 2011; 9: 204.

25. Hersh EM, O'Day SJ, Powderly J, Khan KD, Pavlick AC, Cranmer LD, et al. A phase II multicenter study of ipilimumab with or without dacarbazine in chemotherapy-naive patients with advanced melanoma. Invest New Drugs. 2011; 29: 489-98.

26. Robert C, Thomas L, Bondarenko I, O'Day S, Weber J, Garbe C, et al. Ipilimumab plus dacarbazine for previously untreated metastatic melanoma. N Engl J Med. 2011; 364: 2517-26.

27. Lynch TJ, Bondarenko I, Luft A, Serwatowski P, Barlesi F, Chacko R, et al. Ipilimumab in combination with paclitaxel and carboplatin as first-line treatment in stage IIIB/IV non-small-cell lung cancer: results from a randomized, double-blind, multicenter phase II study. J Clin Oncol. 2012; 30: 2046-54.

28. Reck M, Bondarenko I, Luft A, Serwatowski P, Barlesi F, Chacko R, et al. Ipilimumab in combination with paclitaxel and carboplatin as first-line therapy in extensive-disease-small-cell lung cancer: results from a randomized, double-blind, multicenter phase 2 trial. Ann Oncol. 2013; 24: 75-83.

29. Slovin SF, Higano CS, Hamid O, Tejwani S, Harzstark A, Alumkal JJ, et al. Ipilimumab alone or in combination with radiotherapy in metastatic castration-resistant prostate cancer: results from an open-label, multicenter phase I/II study. Ann Oncol. 2013; 24: 1813-21.

30. Horinouchi H, Yamamoto N, Fujiwara Y, Sekine I, Nokihara H, Kubota K, et al. Phase I study of ipilimumab in phased combination with paclitaxel and carboplatin in Japanese patients with non-small-cell lung cancer. Invest New Drugs. 2015; 33: 881-9.

31. Yamazaki N, Kiyohara Y, Uhara H, Fukushima S, Uchi H, Shibagaki N, et al. Phase II study of ipilimumab monotherapy in Japanese patients with advanced melanoma. Cancer Chemother Pharmacol. 2015; 76: 997-1004.

32. Yamazaki N, Uhara H, Fukushima S, Uchi H, Shibagaki N, Kiyohara Y, et al. Phase II study of the immune-checkpoint inhibitor ipilimumab plus dacarbazine in Japanese patients with previously untreated, unresectable or metastatic melanoma. Cancer Chemother Pharmacol. 2015; 76: 969-75.

33. Amin A, Lawson DH, Salama AK, Koon HB, Guthrie T, Jr., Thomas SS, et al. Phase II study of vemurafenib followed by ipilimumab in patients with previously untreated BRAF-mutated metastatic melanoma. J Immunother Cancer. 2016; 4: 44

34. Eggermont AMM, Chiarion-Sileni V, Grob J-J, Dummer R, Wolchok JD, Schmidt H, et al. Prolonged Survival in Stage III Melanoma with Ipilimumab Adjuvant Therapy. N Engl J Med. 2016; 375: 1845-55. 
35. Puzanov I, Milhem MM, Minor D, Hamid O, Li A, Chen L, et al. Talimogene Laherparepvec in Combination With Ipilimumab in Previously Untreated, Unresectable Stage IIIB-IV Melanoma. J Clin Oncol. 2016; 34: 2619-26.

36. Reck M, Luft A, Szczesna A, Havel L, Kim SW, Akerley W, et al. Phase III Randomized Trial of Ipilimumab Plus Etoposide and Platinum Versus Placebo Plus Etoposide and Platinum in Extensive-Stage Small-Cell Lung Cancer. J Clin Oncol. 2016; 34: 3740-8.

37. Ascierto PA, Del Vecchio M, Robert C, Mackiewicz A, Chiarion-Sileni V, Arance A, et al. Ipilimumab $10 \mathrm{mg} / \mathrm{kg}$ versus ipilimumab $3 \mathrm{mg} / \mathrm{kg}$ in patients with unresectable or metastatic melanoma: a randomised, double-blind, multicentre, phase 3 trial. Lancet Oncol. 2017; 18: 611-22.

38. Bang Y-J, Cho JY, Kim YH, Kim JW, Di Bartolomeo M, Ajani JA, et al. Efficacy of Sequential Ipilimumab Monotherapy versus Best Supportive Care for Unresectable Locally Advanced/Metastatic Gastric or Gastroesophageal Junction Cancer. Clin Cancer Res. 2017; 23: 5671-8.

39. Beer TM, Kwon ED, Drake CG, Fizazi K, Logothetis C, Gravis G, et al. Randomized, Double-Blind, Phase III Trial of Ipilimumab Versus Placebo in Asymptomatic or Minimally Symptomatic Patients With Metastatic Chemotherapy-Naive Castration-Resistant Prostate Cancer. J Clin Oncol. 2017; 35: 40-7.

40. Chesney J, Puzanov I, Collichio F, Singh P, Milhem MM, Glaspy J, et al. Randomized, Open-Label Phase II Study Evaluating the Efficacy and Safety of Talimogene Laherparepvec in Combination With Ipilimumab Versus Ipilimumab Alone in Patients With Advanced, Unresectable Melanoma. J Clin Oncol. 2018; 36: 1658-67.

41. Govindan R, Szczesna A, Ahn MJ, Schneider CP, Gonzalez Mella PF, Barlesi F, et al. Phase III Trial of Ipilimumab Combined With Paclitaxel and Carboplatin in Advanced Squamous Non-Small-Cell Lung Cancer. J Clin Oncol. 2017; 35: 3449-57.

42. Hellmann MD, Rizvi NA, Goldman JW, Gettinger SN, Borghaei H, Brahmer $\mathrm{JR}$, et al. Nivolumab plus ipilimumab as first-line treatment for advanced non-small-cell lung cancer (CheckMate 012): results of an open-label, phase 1, multicohort study. The Lancet Oncology. 2017; 18: 31-41.

43. Schachter J, Ribas A, Long GV, Arance A, Grob JJ, Mortier L, et al. Pembrolizumab versus ipilimumab for advanced melanoma: final overall survival results of a multicentre, randomised, open-label phase 3 study (KEYNOTE-006). Lancet. 2017; 390: 1853-62.

44. Weber J, Mandala M, Del Vecchio M, Gogas HJ, Arance AM, Cowey CL, et al. Adjuvant Nivolumab versus Ipilimumab in Resected Stage III or IV Melanoma. N Engl J Med. 2017; 377: 1824-35.

45. Weide B, Martens A, Wistuba-Hamprecht K, Zelba H, Maier L, Lipp HP, et al. Combined treatment with ipilimumab and intratumoral interleukin-2 in pretreated patients with stage IV melanoma-safety and efficacy in a phase II study. Cancer Immunol Immunother. 2017; 66: 441-9.

46. Klein O, Ebert LM, Nicholaou T, Browning J, Russell SE, Zuber M, et al. Melan-A-specific cytotoxic $\mathrm{T}$ cells are associated with tumor regression and autoimmunity following treatment with anti-CTLA-4. Clin Cancer Res. 2009; 15: 2507-13.

47. Weber J. Ipilimumab: controversies in its development, utility and autoimmune adverse events. Cancer Immunol Immunother. 2009; 58: 823-30.

48. Ji RR, Chasalow SD, Wang L, Hamid O, Schmidt H, Cogswell J, et al. An immune-active tumor microenvironment favors clinical response to ipilimumab. Cancer Immunol Immunother. 2012; 61: 1019-31.

49. Fecher LA, Agarwala SS, Hodi FS, Weber JS. Ipilimumab and its toxicities: a multidisciplinary approach. Oncologist. 2013; 18: 733-43.

50. Martin JAL, Cao MG, Sereno M, Mayordomo J, Hidalgo M, Campos B, et al. Ipilimumab in older patients: spanish melanoma multidisciplinary group (gem) experience in the expanded access programme. Ann Oncol. 2012; 23: 374-5.

51. Lawrence D, McDermott DF, Hamid O, Weber JS, Wolchok JD, Richards J, et al. Ipilimumab (IPI) expanded access program (EAP) for patients (PTS) with stage III/IV melanoma: safety data by subgroups. Ann Oncol. 2012; 23: 368.

52. Bertrand A, Kostine M, Barnetche T, Truchetet ME, Schaeverbeke T. Immune related adverse events associated with anti-CTLA-4 antibodies: systematic review and meta-analysis. BMC Med. 2015; 13: 211.

53. CiRen B, Wang $X$, Long $Z$. The evaluation of immunotherapy and chemotherapy treatment on melanoma: a network meta-analysis. Oncotarget. 2016; 7: 81493-511. 